REVIEW ARTICLE

\title{
Validação de métodos microbiológicos alternativos: uma visão geral
}

\section{Validation of alternative microbiological methods: an overview}

\author{
Francisca Airlane Esteves de Brito1, Lorena Cristina Rodrigues Bezerra², \\ Aíris Maria Araújo Melo ${ }^{3,4}$, Roselayne Ferro Furtado5* (D), Maria de Fátima Borges ${ }^{5}$, \\ Evânia Altina Teixeira de Figueiredo ${ }^{1}$
}

\author{
${ }^{1}$ Universidade Federal de Ceará (UFC), Departamento de Ciência e Tecnologia de Alimentos, Fortaleza/CE - Brasil \\ ${ }^{2}$ Universidade Estadual do Ceará (UECE), Departamento de Química, Fortaleza/CE - Brasil \\ ${ }^{3}$ Universidade Estadual do Ceará (UECE), Rede Nordeste de Biotecnologia, Fortaleza/CE - Brasil \\ ${ }^{4}$ Instituto Federal do Piauí (IFPI), Campus of Picos, Picos/PI - Brasil \\ ${ }^{5}$ Embrapa Agroindústria Tropical, Fortaleza/CE - Brasil
}

${ }^{*}$ Corresponding Author: Roselayne Ferro Furtado, Embrapa Agroindústria Tropical - Pesquisa e

Desenvolvimento, Rua Dra. Sara Mesquita, 2270, Planalto Pici, CEP: 60511-110, Fortaleza/CE - Brasil, e-mail: roselayne.furtado@embrapa.br

Cite as: Brito, F. A. E., Bezerra, L. C. R., Melo, A. M. A., Furtado, R. F., Borges, M. F., \& Figueiredo, E. A. T. (2021). Validation of alternative microbiological methods: an overview. Brazilian Journal of Food Technology, 24, e2020023. https://doi.org/10.1590/1981-6723.02320

\section{Resumo}

O desenvolvimento de novos métodos de análise microbiológica requer uma avaliação da igualdade ou superioridade deste em relação a um método padrão ouro - método rotineiramente utilizado. Este processo de avaliação denomina-se validação. O método é validado se seu desempenho estiver satisfatoriamente de acordo com os parâmetros analisados. Neste trabalho, abordamos os critérios para a validação de novos métodos de análise microbiológica da AOAC, FDA e da ISO 16140

Palavras-chave: Protocolo; Novos métodos; Validação; Contaminação; Qualidade; FDA; AOAC; ISO; Microbiologia.

\begin{abstract}
The development of new methods for microbiological analysis of food requires an assessment of their equality or superiority in relation to a routinely used gold standard method. This evaluation process is called validation. The method is validated if its performance is satisfactorily in accordance with the analyzed parameters. This study address the validation criteria of new microbiological analysis methods developed by AOAC, FDA, and ISO 16140.
\end{abstract}

Keywords: Protocol; New methods; Validation; Contamination; Quality; FDA; AOAC; ISO; Microbiology.

\section{Introdução}

\subsection{Processo de validação}

Em microbiologia, existem os métodos de referência oficiais (padrão ouro) para detecção dos microrganismos, bem como dos seus metabólitos, para os diferentes tipos de amostras. Contudo, muitos deles 
são demorados, envolvem inúmeras etapas e muitas vezes também demandam recurso humano qualificado e infraestrutura laboratorial. A cada dia, surgem mais metodologias e possibilidades de detecção desses analitos com o apelo de serem mais rápidos, práticos, de menor custo e igualmente ou superiores em termos de sensibilidade (Mata et al., 2016; Thakur et al., 2018; Rajapaksha et al., 2019; Cui et al., 2020; Ferone et al., 2020, Kumar et al., 2020; Melo et al., 2020; You et al., 2018; Hao et al., 2020; Jiang et al., 2021; Yang et al., 2021). Todas essas formas de detecção emergentes geralmente resultam em um novo produto comercial na forma de kits ou dispositivos. Estes, antes de alcançarem o mercado, devem ser submetidos a estudos de validação de acordo com alguma normativa reconhecida e aceita pelo órgão regulamentador do País (Brito et al., 2020; Lemos et al., 2020).

Entende-se validação como uma etapa posterior à fase de desenvolvimento, indispensável para assegurar o desempenho funcional e reproduzível de um produto ou processo, visando a uma escala comercial (International Council for Harmonisation of Technical Requirements for Pharmaceuticals for Human Use, 1995; Food and Drug Administration, 2015a, 2018). A validação de um método alternativo para a microbiologia é o processo pelo qual um ou mais laboratórios confirmam, por exame, e fornecem evidências objetivas de que os requisitos para usos específicos são atendidos. Métodos alternativos desenvolvidos como opções a métodos convencionais devem passar impreterivelmente por esta etapa (Jasson et al., 2010). A validação serve para demonstrar que o método pode detectar e identificar um analito ou analitos em uma ou mais matrizes a serem analisadas, usando um ou mais instrumentos ou plataformas, e possui sensibilidade, especificidade, exatidão, veracidade, reprodutibilidade e precisão demonstradas, para assegurar que os resultados sejam significativos e apropriados para a tomada de uma decisão (Food and Drug Administration, 2019; Roshchina \& Gunar, 2020).

Ao longo do tempo, Anvisa e Inmetro, entidades regulatórias do Brasil, têm realizado inúmeras recomendações que servem de guias para validação de métodos analíticos e bioanalíticos (Brasil, 2003, 2012, 2017; Instituto Nacional de Metrologia, Qualidade e Tecnologia, 2016, 2017, 2018). Nelas, são encontradas terminologias rotineiramente usadas em estudos de validação. A especificidade e a seletividade referem-se à capacidade que o método possui de medir exatamente um composto em presença de outros componentes, tais como impurezas, produtos de degradação e componentes da matriz. A precisão é a avaliação da proximidade dos resultados obtidos em uma série de medidas de uma amostragem múltipla de uma mesma amostra. Ela é considerada em três níveis: a) repetibilidade (precisão intracorrida) - concordância entre os resultados dentro de um curto período de tempo com o mesmo analista e a mesma instrumentação; b) precisão intermediária (precisão intercorridas) - concordância entre os resultados do mesmo laboratório, mas obtidos em dias diferentes, com analistas diferentes e/ou equipamentos diferentes; c) reprodutibilidade (precisão interlaboratorial) - concordância entre os resultados obtidos em laboratórios diferentes, como em estudos colaborativos. A compreeensão destes termos ajuda a entender e valorizar as análises exigidas para a validação de métodos.

No Brasil, não existe uma normativa a ser seguida para a validação de novos métodos alternativos de análise microbiológica, sendo recomendada a observação de normativas de caráter internacional estabelecidas por órgãos regulamentadores reconhecidos mundialmente. O Inmetro, em 2017, publicou um documento baseado em diretrizes internacionais que contém aplicações sobre os requisitos de acreditação para ensaios microbiológicos e podem auxiliar laboratórios a demonstrarem um método microbiológico normalizado (Instituto Nacional de Metrologia, Qualidade e Tecnologia, 2017). Neste sentido, no presente artigo, serão abordadas três principais normas de validação para métodos alternativos de análise microbiológica para a área de alimentos, estabelecidos pela AOAC, FDA e ISO.

\subsection{AOAC}

Criada em 1884, inicialmente com o nome Associação Oficial de Químicos, em 1991, passou a ser conhecida simplesmente como AOAC Internacional (Association of Official Analytical Chemists, 2019a). 
A AOAC evoluiu para uma associação independente, terceirizada e não governamental, envolvendo agências governamentais, instituições de pesquisa e cientistas individuais, que trabalham em rede, participam e deliberam sobre métodos e medidas de qualidade (Association of Official Analytical Chemists, 2019b). Ela estabelece normativas para os novos métodos químicos e microbiológicos serem avaliados quanto à confiabilidade. A AOAC possui dois programas pelos quais os métodos são avaliados e aprovados. Esses programas são os OMA (Official Methods of Analysis ${ }^{\mathrm{SM}}$ ) e PTM (Performance Tested Methods). O programa OMA é internacionalmente conhecido por seu rigoroso exame científico e sistemático dos Métodos Oficiais de Análise resultantes. O programa PTM evoluiu para um programa de certificação de métodos de métodos alternativos. O programa PTM oferece certificação como um ponto final para avaliação de método ou como entrada para validação de método para programas que exigem maior confiança e informações de reprodutibilidade do método.

Neste artigo, será feita uma abordagem segundo AOAC - OMA. Se possível, os métodos alternativos microbiológicos devem ser comparados a um método de cultura de referência. Os métodos alternativos podem ser qualitativos ou quantitativos na $\mathrm{AOAC}$ e os protocolos são divididos em estudos pré-colaborativo e colaborativo (Association of Official Analytical Chemists, 2012).

Estudo pré-colaborativo precede o estudo Interlaboratorial, denominado também de estudo colaborativo interlaboratorial ou simplesmente colaborativo. O objetivo de um estudo pré-colaborativo é demonstrar a aplicabilidade do método a uma ou várias categorias de alimentos. O objetivo do Estudo Colaborativo é fornecer uma estimativa realista dos atributos de um método, particularmente desvios sistemáticos e aleatórios, esperados quando o método é usado na prática real. Nestes estudos, os alimentos são comumente inoculados com o organismo de interesse quando os produtos naturalmente contaminados são pouco frequentes. Geralmente, um isolado, uma cepa, um sorotipo ou uma espécie diferente é usado para cada tipo de alimento. A inoculação do produto deve ser conduzida com uma cultura pura de uma estirpe. Culturas mistas não são recomendadas (Feldsine et al., 2002).

Para os métodos qualitativos realizados sob o aspecto pré-colaborativo, cada tipo de alimento é dividido em dois níveis. Um serve como controle negativo e outro seria um nível fracionário de inoculação. As amostras de teste controle e inoculadas devem ser preparadas ao mesmo tempo. Dependendo do laboratório em satisfazer este critério de validação, pode ser aconselhável preparar um terceiro nível que tenha um alto nível de inóculo, mas é opcional. O baixo nível de inóculo é estabelecido com base no limite de detecção do método testado, como, por exemplo, porção de teste de 1 a $5 \mathrm{UFC} / 25 \mathrm{~g}$. O alto nível de inóculo é fixado em 10 a 50 UFC/porção de teste de $25 \mathrm{~g}$. Em nível colaborativo, o terceiro nível (alto nível de inóculo) passa a ser compulsório. As amostras de controle não precisam ser incluídas nos tipos de alimentos naturalmente contaminados (Feldsine et al., 2002).

Para os métodos quantitativos, sejam eles pré- colaborativo ou colaborativo, os alimentos são artificialmente contaminados e divididos em três níveis inoculados (alto, médio e baixo). O nível mais baixo deve aproximar-se do limite de detecção do método alternativo e os níveis médio e alto podem ser aproximadamente um a dois logaritmos mais altos, respectivamente. Amostras de controle não inoculadas também são incluídas para cada tipo de alimento inoculado (Feldsine et al., 2002).

A quantificação do número mais provável (NMP) dos níveis de contaminação é realizada no laboratório responsável no dia em que a análise das amostras de teste é iniciada. Os NMPs são conduzidos de acordo com o método de cultura de referência especificado no protocolo.

Seis porções de teste por nível de analito para cada tipo de alimento e seis porções de controle negativas (não inoculadas) para cada tipo de alimento são requeridos. As porções de teste não devem ser identificadas quando enviadas para laboratórios participantes das análises (Feldsine et al., 2002).

Microrganismos competidores podem ser analisados com o intuito de simular mais de perto as condições encontradas na natureza. Os níveis de contaminação do competidor, que podem ocorrer naturalmente ou introduzidos artificialmente, devem ser pelo menos um logaritmo superior ao microrganismo alvo. A partir 
destas análises, pode-se ter noção da inclusividade ou exclusividade. Inclusividade ou sensibilidade é a capacidade do método alternativo para detectar o analito alvo na presença de outras cepas. Exclusividade ou especificidade é a falta de interferência no método alternativo na presença de cepas não alvo, que são potencialmente reativas de forma cruzada.

No Estudo Colaborativo para métodos qualitativos, é necessário um mínimo de 10 conjuntos de dados laboratoriais válidos por tipo de alimento. O planejamento deve incluir pelo menos 12-15 laboratórios por alimento porque alguns laboratórios são geralmente eliminados por várias razões. No método quantitativo, são necessários pelo menos oito laboratórios. Em casos especiais, envolvendo equipamentos muito caros ou laboratórios especializados, o estudo pode ser realizado com um mínimo de cinco laboratórios.

O número de diferentes categorias de alimentos para os testes pré-colaborativos e colaborativos depende da aplicabilidade do método. No pré-colaborativo, devem-se analisar pelo menos vinte tipos diferentes de alimentos. No colaborativo, se o método é específico para apenas uma categoria de alimento (por exemplo, detecção de Campylobacter em ostras), apenas um tipo de alimento deve ser incluído. Se a aplicabilidade é mais ampla (por exemplo, a detecção de Salmonella em todos os alimentos), então seis categorias de alimentos devem ser incluídas.

Os programas da AOAC têm os seguintes indicadores de desempenho para métodos qualitativos: taxa de falsos positivos, taxa de falsos negativos, sensibilidade e especificidade (Feldsine et al., 2002). Para os métodos quantitativos, os indicadores são outros: repetibilidade, reprodutibilidade e desvio padrão relativo (Tabela 1).

Tabela 1. Indicadores de desempenho da AOAC para métodos qualitativos e quantitativos.

\begin{tabular}{|c|c|}
\hline \multicolumn{2}{|r|}{ Métodos qualitativos } \\
\hline Indicador & Definição \\
\hline Taxa de falsos positivos & $\begin{array}{c}\text { Probabilidade de uma amostra negativa pelo método de referência ser detectada como } \\
\text { positiva pelo método teste, isto é, número de amostras positivas pelo método teste e } \\
\text { negativas pelo método de referência / no total de amostras negativas pelo método de } \\
\text { referência. }\end{array}$ \\
\hline Taxa de falsos negativos & $\begin{array}{c}\text { É a probabilidade de uma amostra positiva pelo método de referência ser detectada como } \\
\text { negativa pelo método teste, isto é, número de amostras negativas pelo método teste e } \\
\text { positivas pelo método de referência / no total de amostras positivas pelo método de } \\
\text { referência. }\end{array}$ \\
\hline Sensibilidade & $\begin{array}{l}\text { Número total de porções do teste positivas confirmadas pelo método dividido pelo número } \\
\text { total de porções de teste positivas confirmadas pelos métodos alternativos e de referência. }\end{array}$ \\
\hline Especificidade & $\begin{array}{l}\text { Número total de porções de teste negativas analisadas pelo método dividido pelo número } \\
\text { total de porções de teste negativas confirmadas pelos métodos alternativos e de referência }\end{array}$ \\
\hline \multicolumn{2}{|r|}{ Métodos quantitativos } \\
\hline Repetibilidade & $\begin{array}{l}\text { A repetibilidade está dentro da precisão do laboratório, designada como a concordância } \\
\text { entre resultados sucessivos e independentes obtidos pelo mesmo método em material de } \\
\text { teste idêntico, sob as mesmas condições (por exemplo, aparelho, operador, laboratório e } \\
\text { tempo de incubação). }\end{array}$ \\
\hline Reprodutibilidade & $\begin{array}{l}\text { A reprodutibilidade está entre a precisão dos laboratórios ou a concordância entre os } \\
\text { resultados dos testes individuais em material de teste idêntico usando o mesmo método e } \\
\text { obtidos por operadores em diferentes laboratórios usando diferentes equipamentos. }\end{array}$ \\
\hline Desvio padrão relativo & $\begin{array}{l}\text { O desvio padrão relativo é uma medida útil de precisão em estudos quantitativos. O desvio } \\
\text { padrão relativo é calculado dividindo a repetibilidade ou a reprodutibilidade pela média. Os } \\
\text { valores do desvio padrão relativo são independentes da quantidade de analito em um } \\
\text { intervalo razoável e facilitam a comparação de variabilidades em diferentes concentrações. }\end{array}$ \\
\hline
\end{tabular}

Fonte: Association of Official Analytical Chemists (2012); Feldsine et al. (2002). 


\subsection{FDA}

O FDA é uma agência do Departamento de Saúde e Serviços Humanos dos Estados Unidos. É responsável por supervisionar a segurança de alimentos nacionais e importados, através de programas de monitoramento de patógenos, toxinas naturais, pesticidas e outros contaminantes; avaliar o potencial de exposição e riscos, e fornecer informações sobre a prevenção de doenças transmitidas por alimentos. Na validação de métodos microbiológicos alternativos para alimentos, três níveis são definidos e servem para demonstrar que o método pode detectar, identificar e, quando aplicável, quantificar um analito ou analitos para um padrão definido de desempenho. $\mathrm{O}$ escalonamento de critérios dentro do processo de validação também fornece características da utilidade do método e direciona para o uso pretendido. Os níveis de validação estão descritos abaixo, de acordo com Food and Drug Administration (2015b).

\subsubsection{Uso de emergência (Nível Um)}

Este nível é o inicial, em termos de validação. É recomendado para casos de emergência. São os métodos desenvolvidos ou modificados para a deteç̧ão de um analito ou uma matriz que não tenha sido previamente reconhecida ou identificada como uma ameaça à segurança dos alimentos ou à saúde pública. Uma vez que a necessidade de uso do método tenha passado e tenha sido determinado que há necessidade de prosseguir a validação, os procedimentos descritos para os níveis subsequentes devem ser seguidos.

O desempenho do método neste nível determinará, em parte, se a validação adicional é útil ou justificada. Todo o trabalho deve ser feito por um ou mais laboratórios. Sensibilidade e especificidade (inclusão e exclusividade) devem ser testadas e incluir um número limitado de cepas (Tabela 2).

Tabela 2. Guia geral para a validação de métodos de identificação para analitos microbianos, segundo FDA.

\begin{tabular}{|c|c|c|c|}
\hline \multicolumn{4}{|c|}{ Processo de validação sem emergência } \\
\hline Critério & $\begin{array}{l}\text { Estudo de validação em } \\
\text { laboratório único }\end{array}$ & $\begin{array}{l}\text { Estudo de validação em } \\
\text { laboratório independente }\end{array}$ & $\begin{array}{l}\text { Estudo de validação } \\
\text { colaborativa }\end{array}$ \\
\hline Laboratório participante & Laboratório de origem & Laboratório colaborador & Laboratórios colaboradores \\
\hline Inclusividade* & $\begin{array}{c}\geq 50 \text { (menos que } 50 \text { não é } \\
\text { válido) (amostras deveriam } \\
\text { ser misturadas e não } \\
\text { identificadas }\end{array}$ & $\begin{array}{c}1 \text { (amostra deveria ser } \\
\text { misturada e não } \\
\text { identificada) }\end{array}$ & $\begin{array}{c}12 \text { (amostras deveriam ser } \\
\text { misturadas e não } \\
\text { identificadas) }\end{array}$ \\
\hline Exclusividade* & $\geq 30$ variedades & 1 & 12 \\
\hline $\begin{array}{l}\text { Diferentes laboratórios } \\
\text { fornecendo dados }\end{array}$ & 1 & 1 & 10 \\
\hline Réplicas & 3 & 3 & 3 \\
\hline $\begin{array}{c}\text { Comparação com método } \\
\text { de referência }\end{array}$ & $\operatorname{sim}$ & $\operatorname{sim}$ & $\operatorname{sim}$ \\
\hline
\end{tabular}

*103 UFC/mL para microrganismos alvo e não alvos crescidos em meio não seletivo. Fonte: Food and Drug Administration (2015).

\subsubsection{Validação em laboratório único (Nível Dois - Parte a)}

Este nível é considerado não emergencial. Um laboratório faz um estudo inicial mais abrangente com níveis definidos de inclusão / exclusividade, conforme mostrado na Tabela 2. Este é o primeiro passo no processo de validação de métodos projetados para regulamentações de análises de rotina. Uma comparação será feita com um método de referência existente. Taxas de falsos positivos levemente mais altas podem ser aceitáveis, uma vez que todas as amostras analisadas exigirão testes de confirmação. 


\subsubsection{Validação em laboratório independente (Nível Dois - Parte b)}

Um laboratório independente participa do estudo de validação utilizando o método do laboratório de origem e os critérios descritos na Tabela 2.

\subsection{Validação colaborativa (Nível Dois - Parte c)}

Este tipo de estudo é aceitável para utilização em qualquer e todas as circunstâncias reguladoras, seja para as análises confirmatórias; investigações de surtos / vigilância, e suporte de conformidade. Um estudo colaborativo é um estudo interlaboratorial em que cada laboratório utiliza o método de análise definido para analisar porções idênticas de materiais homogêneos, para avaliar as características de desempenho obtidas para esse método de análise.

Os critérios contidos nas tabelas para métodos qualitativos e quantificáveis descrevem os requisitos a serem executados pelos laboratórios de origem e de colaboração, respectivamente.

Da mesma forma que é estabelecido pela AOAC, nos protocolos do FDA para os métodos qualitativos e quantitativos, os critérios de avaliação são bem parecidos, existindo pequenas diferenças nas quantidades de níveis de inóculo e de repetições por alimento, em cada nível do inóculo testado.

Os indicadores de desempenho utilizados para os métodos de identificação microbiológicos são: acurácia (proporção de amostras positivas verdadeiras recuperadas do novo teste em comparação com o teste de referência, quando inoculadas de forma semelhante), taxa de falso positivo, taxa de falso negativo, inclusividade, especificidade, limite de detecção, reprodutibilidade, repetibilidade e robustez (determina se pequenas alterações no procedimento ou fatores ambientais influenciam o desempenho do teste) (Food and Drug Administration, 2015b).

\subsection{ISO 16140}

ISO (International Organization for Standardization) 16140: 2016 especifica o princípio geral e o protocolo técnico para a validação de métodos microbiológicos de produção dos alimentos (International Organization for Standardization, 2016a). Trata-se de uma normativa, não disponibilizada gratuitamente na internet, ao contrário da AOAC e do FDA, e serve de documento orientador e regulador de métodos de gêneros alimentícios dentro da União Europeia e para importação. O documento é revisado a cada cinco anos. A ISO 16140 encontra-se dividida nas seguintes partes: a) Parte 1: Vocabulário - (International Organization for Standardization, 2016b); b) Parte 2: Protocolo para a validação de métodos alternativos com um método de referência (certificação) (International Organization for Standardization, 2016c); c) Parte 3: Protocolo para a verificação de métodos alternativos de referência e validados implementados em um único laboratório (acreditação) (International Organization for Standardization, 2016d); d) Parte 4: Protocolo para validação de método em laboratório único (interno)(acreditação) (International Organization for Standardization, 2016e); e) Parte 5: Protocolo para validação interlaboratorial fatorial de métodos não alternativos (acreditação) (International Organization for Standardization, 2016f), e f) Parte 6: Protocolo para a validação de métodos alternativos (proprietários) para confirmação microbiológica e procedimentos de tipagem (certificação) (International Organization for Standardization, 2016g).

Destina-se a produtos direcionados ao consumo humano, à alimentação animal, a amostras ambientais na área de produção de alimentos e rações, ao manuseio e às amostras do estágio primário de produção. A normativa ISO 16140 é particularmente aplicável à determinação de bactérias e fungos. Algumas cláusulas da ISO 16140 poderiam ser aplicáveis a outros microrganismos ou seus metabólitos, a depender de caso a caso. No futuro, a orientação para outros organismos (por exemplo, vírus e parasitas) está prevista para ser incluída nesta parte ou em uma parte separada da ISO 16140. 
O protocolo de validação consiste em duas fases: a) estudo comparativo do método alternativo com um método de referência conduzido no laboratório responsável e b) um estudo interlaboratorial que também pode ser conduzido paralelamente (International Organization for Standardization, 2016a).

Para métodos quantitativos, na fase de estudo comparativo, são utilizados quatro indicadores: exatidão, limite de detecção / quantificação e inclusão / exclusividade. Na fase de estudo interlaboratorial, é abordada a precisão interlaboratorial do método alternativo contra o método de referência, estimando os indicadores de repetibilidade (variabilidade aleatória mínima), reprodutibilidade (variabilidade aleatória máxima) e erro sistemático. Esta fase deve envolver pelo menos oito laboratórios e as amostras são preparadas a partir de uma matriz, contaminadas em quatro níveis, incluindo um controle negativo. A análise deve ser conduzida em duplicata pelo método alternativo e o de referência (Lombard \& Leclercq, 2011).

Para os métodos qualitativos, são três os indicadores: exatidão, limite de detecção e inclusão / exclusividade, de modo a caracterizar o desempenho do método alternativo em relação ao método de referência. Na segunda fase, o estudo interlaboratorial requer pelo menos dez laboratórios colaborativos. As amostras são preparadas com uma matriz contaminada em três níveis (incluindo um controle negativo) (Lombard \& Leclercq, 2011). Os resultados são comparados aos obtidos quanto aos indicadores na primeira fase de estudo colaborativo.

\section{Conclusão}

A validação dos novos métodos alternativos é estritamente necessária para garantir aos laboratórios e seus clientes (indústria de alimentos, autoridades de controle público) que os resultados obtidos com estes métodos são válidos e possuem desempenho equivalente ou melhor do que aqueles obtidos com o correspondente de referência, os chamados "padrões ouro".

Os protocolos de validação apresentam muitos pontos em comum e por isso muitas vezes são denominados de harmonizados. Os protocolos de FDA e AOAC são mais acessíveis, no sentido de que podem ser obtidos gratuitamente nos seus respectivos endereços eletrônicos.

\section{Referências}

Association of Official Analytical Chemists - AOAC. (2012). OAC INTERNATIONAL Methods committee guidelines for validation of microbiological methods for food and environmental surfaces. Rockville: AOAC. Recuperado em 19 de julho de 2021, de https://members.aoac.org/AOAC_Docs/StandardsDevelopment/AOAC_Validation_Guidelines_for_Food_MicrobiologyPrepub_version.pdf

Association of Official Analytical Chemists - AOAC. (2019a). AOAC's name and what does it stand for? Rockville: AOAC. Recuperado em 3 de fevereiro de 2020, de http://www.aoac.org/aoac_prod_imis/AOAC_Member/About_AOAC/Company_Overview/History.aspx?WebsiteKey=2e25ab5a1f6d-4d78-a498-19b9763d11b4

Association of Official Analytical Chemists - AOAC. (2019b). Official methods of analysis. Rockville: AOAC. Recuperado em 3 de fevereiro de 2020, de http://eoma.aoac.org/aboutaoac.asp

Brasil. Agência Nacional de Vigilância Sanitária - ANVISA. (2003, junho 2). Determina a publicação do "Guia para validação de métodos (Resolução RE n 899, de 29 de maio de 2003). Diário Oficial [da] República Federativa do Brasil, Brasília. Recuperado em 3 de fevereiro de 2020, de http://bvsms.saude.gov.br/bvs/saudelegis/anvisa/2003/res0899_29_05_2003.html

Brasil. Agência Nacional de Vigilância Sanitária - ANVISA. (2012, maio 22). Dispõe sobre os requisitos mínimos para a validação de métodos bioanalíticos empregados em estudos com fins de registro e pós-registro de medicamentos (Resolução RDC $n^{\circ} 27$, de 17 de maio de 2012). Diário Oficial [da] República Federativa do Brasil, Brasília. Recuperado em 3 de fevereiro de 2020, de http://bvsms.saude.gov.br/bvs/saudelegis/anvisa/2012/rdc0027_17_05_2012.html

Brasil. Agência Nacional de Vigilância Sanitária - ANVISA. (2017, julho 25). Dispõe sobre a validação de métodos analíticos e dá outras providências (Resolução RDC n 166, de 24 de julho de 2017). Diário Oficial [da] República Federativa do Brasil, Brasília. Recuperado em 3 de fevereiro de 2020, de

http://antigo.anvisa.gov.br/documents/10181/2721567/RDC_166_2017_COMP.pdf/d5fb92b3-6c6b-4130-8670-4e3263763401

Brito, F. A. E., Bezerra, C. R., Furtado, R. F., Melo, A. M. A., Machado, T. F., Oliveira, M. A., \& Figueiredo, E. A. O. (2020).

Precollaborative validation of an amperometric immunosensor for Salmonella. Proceedings, 4 ,

http://dx.doi.org/10.3390/IECB2020-07031 
Cui, F., Ye, Y., Ping, J., \& Sun, X. (2020). Carbon dots: Current advances in pathogenic bacteria monitoring and prospect applications. Biosensors \& Bioelectronics, 156, 112085. PMid:32275580. http://dx.doi.org/10.1016/j.bios.2020.112085

Feldsine, P., Abeyta, C., \& Andrews, W. H. (2002). AOAC International Methods Committee Guidelines for Validation of Qualitative and Quantitative Food Microbiological Official Methods of Analysis. Journal of AOAC International, 85(5), 1187-1200. PMid:12374420. http://dx.doi.org/10.1093/jaoac/85.5.1187

Ferone, M., Gowen, A., Fanning, S., \& Scannell, A. G. M. (2020). Microbial detection and identification methods: Bench top assays to omics approaches. Comprehensive Reviews in Food Science and Food Safety, 19(6), 3106-3129. PMid:33337061. http://dx.doi.org/10.1111/1541-4337.12618

Food and Drug Administration - FDA. (2015). Guidelines for the validation of analytical methods for the detection of microbial pathogens in foods and feeds. Silver Spring: FDA. Recuperado em 3 de fevereiro de 2020, de https://www.fda.gov/media/83812/download

Food and Drug Administration - FDA. (2018). Bioanalytical method validation guidance for industry. Silver Spring: FDA. Recuperado em 3 de fevereiro de 2020, de https://www.fda.gov/media/70858/download

Food and Drug Administration - FDA. (2019). Guidelines for the validation of analytical methods for the detection of microbial pathogens in foods and feeds (3rd ed.). Silver Spring: FDA. Recuperado em 3 de fevereiro de 2020, de https://www.fda.gov/media/83812/download

Hao, Z., Lin, X., Li, J., Yin, Y., Gao, X., Wang, S., \& Liu, Y. (2020). Multifunctional nanoplatform for dual-mode sensitive detection of pathogenic bacteria and the real-time bacteria inactivation. Biosensors \& Bioelectronics, 173, 112789. PMid:33220533. http://dx.doi.org/10.1016/j.bios.2020.112789

Instituto Nacional de Metrologia, Qualidade e Tecnologia - Inmetro. (2016). Orientação sobre validação de métodos analíticos. Recuperado em 3 de fevereiro de 2020, de http://www.inmetro.gov.br/Sidoq/Arquivos/Cgcre/DOQ/DOQ-Cgcre-8_05.pdf

Instituto Nacional de Metrologia, Qualidade e Tecnologia - Inmetro. (2017). DOQ-CGCRE-089: Orientação sobre avaliação de desempenho de métodos analíticos: Microbiologia. Recuperado em 3 de fevereiro de 2020, de http://www.inmetro.gov.br/credenciamento/organismos/doc_organismos.asp?torganismo=calibensaios

Instituto Nacional de Metrologia, Qualidade e Tecnologia - Inmetro. (2018). DOQ-CGCRE-008: Orientação sobre validação de métodos analíticos. Recuperado em 3 de fevereiro de 2020, de http://www.inmetro.gov.br/credenciamento/organismos/doc_organismos.asp?torganismo=calibensaios

International Council for Harmonisation of Technical Requirements for Pharmaceuticals for Human Use - ICH. (1995). Validation of analytical procedures: Definitions and terminology. Recuperado em 3 de fevereiro de 2020, de https://www.ema.europa.eu/en/ich-q2-r1-validation-analytical-procedures-text-methodology

International Organization for Standardization - ISO. (2016a). ISO 16140: Microbiology of food and animal feeding stuffsprotocol for the validation of alternative methods. Geneva: ISO. Recuperado em 3 de fevereiro de 2020, de https://www.sis.se/api/document/preview/903465/

International Organization for Standardization - ISO. (2016b). ISO 16140-1: Microbiology of the food chain: Method validation: Part 1: Vocabulary. Geneva: ISO. Recuperado em 3 de fevereiro de 2020, de https://www.iso.org/standard/54869.html

International Organization for Standardization - ISO. (2016c). ISO 16140-2: Microbiology of the food chain: Method validation Part 2: Protocol for the validation of alternative (proprietary) methods against a reference method. Geneva: ISO. Recuperado em 3 de fevereiro de 2020, de https://www.iso.org/standard/54870.html

International Organization for Standardization - ISO. (2016d). ISO/DIS 16140-3: Microbiology of the food chain: Method validation: Part 3: Protocol for the verification of reference and validated alternative methods implemented in a single laboratory. Geneva: ISO. Recuperado em 3 de fevereiro de 2020, de https://www.iso.org/standard/66324.html

International Organization for Standardization - ISO. (2016e). ISO/FDIS 16140-4: Microbiology of the food chain: Method validation: Part 4: Protocol for method validation in a single laboratory. Geneva: ISO. Recuperado em 3 de fevereiro de 2020, de https://www.iso.org/standard/66325.html

International Organization for Standardization - ISO. (2016f). ISO/FDIS 16140-5: Microbiology of the food chain: Method validation: Part 5: Protocol for factorial interlaboratory validation for non-proprietary methods. Geneva: ISO. Recuperado em 3 de fevereiro de 2020, de https://www.iso.org/standard/66326.html

International Organization for Standardization - ISO. (2016g). ISO/FDIS 16140-6: Microbiology of the food chain: Method validation: Part 6: Protocol for the validation of alternative (proprietary) methods for microbiological confirmation and typing procedures. Geneva: ISO. Recuperado em 3 de fevereiro de 2020, de https://www.iso.org/standard/66327.html

Jasson, V., Jacxsens, L., Luning, P., Rajkovic, A., \& Uyttendaele, M. (2010). Alternative microbial methods: An overview and selection criteria. Food Microbiology, 27(6), 710-730. PMid:20630313. http://dx.doi.org/10.1016/j.fm.2010.04.008

Jiang, H., Sun, Z., Guo, Q., \& Weng, X. (2021). Microfluidic thread- based electrochemical aptasensor for rapid detection of Vibrio parahaemolyticus. Biosensors \& Bioelectronics, 182, 113191. PMid:33780852.

http://dx.doi.org/10.1016/j.bios.2021.113191

Kumar, N., Wang, W., Ortiz-Marquez, J. C., Catalano, M., Gray, M., Biglari, N., Hikari, K., Ling, X., Gao, J., van Opijnen, T., \& Burch, K. S. (2020). Dielectrophoresis assisted rapid, selective and single cell detection of antibiotic resistant bacteria with GFETs. Biosensors \& Bioelectronics, 156, 112123. PMid:32174552. http://dx.doi.org/10.1016/j.bios.2020.112123

Lemos, M. A., Ghisleni, D. D. M., \& Pinto, T. de J.A. (2020). Validation of alternative microbiological method in non-sterile pharmaceutical product through the reference strains and productive environment bioburden. journal of pharmaceutical innovation, 15(4), 641-650. http://dx.doi.org/10.1007/s12247-019-09408-1 
Lombard, B., \& Leclercq, A. (2011). Validation of innovative food microbiological methods according to the EN ISO 16140 standard. Food Analytical Methods, 4(2), 163-172. http://dx.doi.org/10.1007/s12161-010-9154-4

Mata, G. M. S. C., Martins, A., Machado, S. G., Pinto, M. S., Carvalho, A. F., \& Vanetti, M. C. (2016). Performance of two alternative methods for Listeria detection throughout Serro Minas cheese ripening. Brazilian Journal of Microbiology, 47(3), 749756. PMid:27268116. http://dx.doi.org/10.1016/j.bjm.2016.04.006

Melo, A. M. A., Oliveira, M. R. F., Ferro-Furtado, R., Borges, M. F., Alves, C. R., Biswas, A., \& Cheng, H. N. (2020). Preparation and characterization of carboxymethyl cashew gum grafted with immobilized antibody for potential biosensor application. Carbohydrate Polymers, 228, 115408. PMid:31635742. http://dx.doi.org/10.1016/j.carbpol.2019.115408

Rajapaksha, P., Elbourne, A., Gangadoo, S., Brown, R., Cozzolino, D., \& Chapman, J. (2019). A review of methods for the detection of pathogenic microorganisms. Analyst, 144(2), 396-411. PMid:30468217. http://dx.doi.org/10.1039/C8AN01488D

Roshchina, M. V., \& Gunar, O. V. (2020). Alternative microbiological methods for drug quality testing and their implementation in pharmacy practice. Pharmaceutical Chemistry Journal, 54(8), 834-837. http://dx.doi.org/10.1007/s11094-020-02283-y

Thakur, B., Zhou, G., Chang, J., Pu, H., Jin, B., Sui, X., Yan, X. C., \& Yang, C.-H. (2018). Rapid detection of single E. coli bacteria using a graphene-based field-effect transistor device. Biosensors \& Bioelectronics, 110, 16-22. PMid:29579645. http://dx.doi.org/10.1016/j.bios.2018.03.014

Yang, E., Li, D., Yin, P., Xie, Q., Li, Y., Qingyu, L., \& Duan, Y. (2021). A novel surface-enhanced Raman scattering (SERS) strategy for ultrasensitive detection of bacteria based on three-dimensional (3D) DNA walker. Biosensors \& Bioelectronics, 172 , 112758. PMid:33157406. http://dx.doi.org/10.1016/j.bios.2020.112758

You, Y., Lim, S., Hahn, J., Choi, Y. C., \& Gunasekaran, S. (2018). Bifunctional linker-based immunosensing for rapid and visible detection of bacteria in real matrices. Biosensors \& Bioelectronics, 100, 389-395. PMid:28954255.

http://dx.doi.org/10.1016/j.bios.2017.09.033 